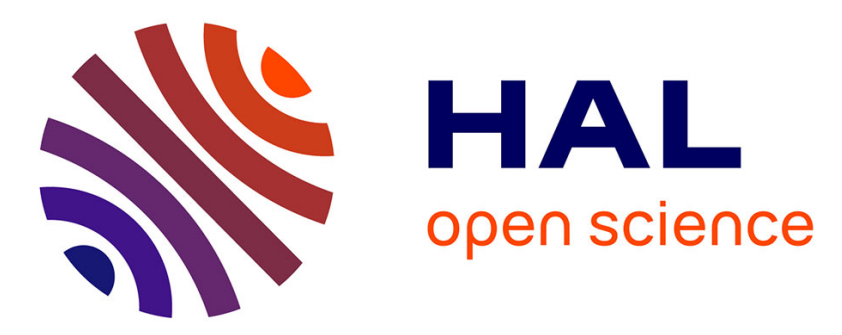

\title{
Shear flow induced transition from liquid-crystalline to polymer behavior in side-chain liquid crystal polymers
}

Laurence Noirez, A. Lapp

\section{To cite this version:}

Laurence Noirez, A. Lapp. Shear flow induced transition from liquid-crystalline to polymer behavior in side-chain liquid crystal polymers. Physical Review Letters, 1997, 78 (1), pp.70-73. 10.1103/PhysRevLett.78.70 . hal-01361942

\section{HAL Id: hal-01361942 \\ https://hal.science/hal-01361942}

Submitted on 7 Sep 2016

HAL is a multi-disciplinary open access archive for the deposit and dissemination of scientific research documents, whether they are published or not. The documents may come from teaching and research institutions in France or abroad, or from public or private research centers.
L'archive ouverte pluridisciplinaire HAL, est destinée au dépôt et à la diffusion de documents scientifiques de niveau recherche, publiés ou non, émanant des établissements d'enseignement et de recherche français ou étrangers, des laboratoires publics ou privés. 


\title{
Shear Flow Induced Transition from Liquid-Crystalline to Polymer Behavior in Side-Chain Liquid Crystal Polymers
}

\author{
L. Noirez and A. Lapp \\ Laboratoire Léon Brillouin (CEA-CNRS), Ce-Saclay 91191, Gif-sur-Yvette, France
}

(Received 9 September 1996)

\begin{abstract}
We determine the structure and conformation of side-chain liquid-crystalline polymers subjected to shear flow in the vicinity of the smectic phase by neutron scattering on the velocity gradient plane. Below the nematic-smectic transition we observe a typical liquid-crystal behavior; the smectic layers slide, leading to a main-chain elongation parallel to the velocity direction. In contrast, a shear applied above the transition induces a tilted main-chain conformation which is typical for polymer behavior. [S0031-9007(96)01984-9]
\end{abstract}

PACS numbers: $61.41 .+\mathrm{e}, 47.50 .+\mathrm{d}, 64.70 . \mathrm{Md}, 83.50 . \mathrm{Ax}$

The effect of shear constraint on the behavior of liquid crystals or on the behavior of "ordinary" polymers has been studied for more than two decades both experimentally and theoretically [1]. In contrast, the investigation of liquid crystal polymer behavior under shear flow has only recently begun [2]. Very interesting behavior can be expected for liquid crystal polymers owing to the competition between the internal strain produced by the side-chain mesogens on the main chain and the external strain brought by the shear process. Recently, we have studied the evolution of the main-chain conformation in the shear plane of a liquid crystal polymethacrylate as a function of the shear rate [3]. It is shown that macroscopic shear is transmitted at a microscopic level by the smectic layers, ensuring an efficient shear of the polymer main chains. The outcome is a macroscopic orientation of the smectic monodomains whose smectic planes are established parallel to the shear plane. The polymer main chains already confined by the mesogenic layers become more elongated along the velocity direction with increasing shear (Fig. 1). These results were obtained from measurements carried out in situ in the shear plane, whereas other planes were observed on a quenched sample after shearing. In this article we consider measurements made in situ in the plane of the velocity gradient (vorticity plane). This plane is particularly interesting since it allows the simultaneous observation of the formation of the smectic phase and of the conformation of the polymer main chain versus shear rate. Such a direct study has never been carried out in bulk, even for nonliquid crystalline polymers. This last experiment [4] confirmed the theory that bulk polymer conformation under shear flow is a tilted elongated shape resulting from a combination of rotational and translational motions. We obtained the same result using our cell with a polystyrene melt.

The liquid-crystalline polymer $\mathrm{PMA}-\mathrm{OC}_{4} \mathrm{H}_{9}$ used here is the same as described in Ref. [2] and corresponds to the structural formula

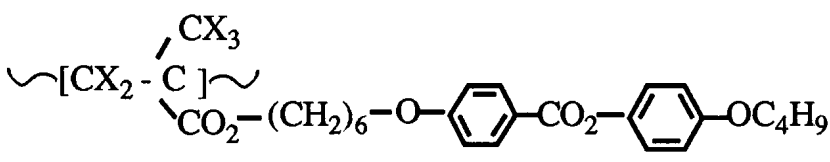

with either $X=H$ or $X=D$ (we used a 1:1 isotopic mixture in order to obtain the central scattering associated with the main-chain conformation).

The sample has the following mesophases and transition temperatures: $T_{g}-35^{\circ} \mathrm{C}-S A 1$ (smectic) $-99^{\circ} \mathrm{C}-\mathrm{N}$ (nematic) $-104{ }^{\circ} \mathrm{C}-I$ (isotropic) (values determined by differential scanning calorimetry with a cooling rate of $\left.-5{ }^{\circ} \mathrm{C} / \mathrm{min}\right)$.

Before and after the shear experiment, gel permeation chromatography provided the same weight average molecular weight $M_{w}=140000$ with polydispersity $I \approx$ 2.8. In contrast with Ref. [2], no significant change of molecular weight was observed. The device is illustrated in Fig. 2. The cell takes advantage of the extremely long mean free path specific of cold neutrons (consequently, such a cell cannot be used for observing the vorticity plane in x-ray measurements). It consists of a thin hollow open aluminum ring ( $3.5 \mathrm{~mm}$ wide, $1 \mathrm{~mm}$ deep) in which the polymer is deposed. This part is static. Above this ring a filled ring of the same width and diameter is placed in contact with the polymer and shear is enabled by a steady-state rotation around the same axis. The cell is tilted at $2^{\circ}-3^{\circ}$ (to the horizontal) in order to place only one side of the ring on the beam trajectory. Small resistors are used as heating devices. The scattered beam is collected on a plane of an $x y$ multidetector (PAXY of

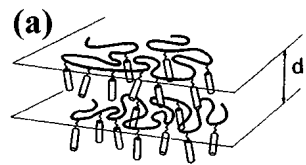

(b)

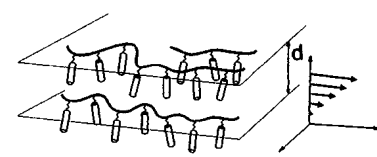

FIG. 1. Main-chain/mesogen organization at rest in the smectic phase. (b) Shear flow resulting conformation in the smectic phase. 


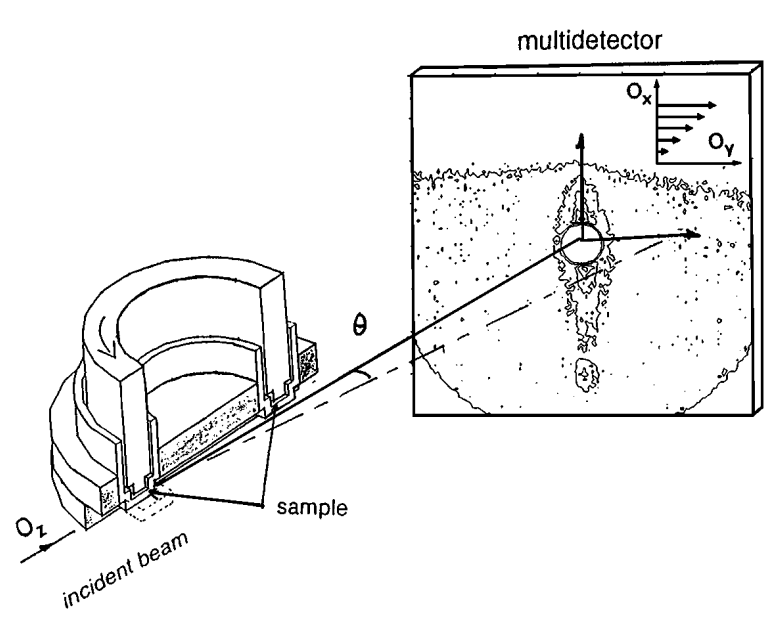

FIG. 2. Schematic of the neutron scattering cell used for the observation of the vorticity plane with a typical neutron scattering pattern $(\lambda=3.5 \AA, d=2 \mathrm{~m})$ obtained with the $\mathrm{H} / \mathrm{D}$ mixture in the smectic phase $\left(T=93{ }^{\circ} \mathrm{C}\right)$ under a shear flow of $6 \mathrm{~s}^{-1}$. The upper part of the pattern corresponds to a shadow due to the upper rotating ring.

the Laboratoire Léon Brillouin) of $128 \times 128$ cells. The data are normalized and the background noise removed. We have used two configurations, depending on the neutron wavelength $\lambda=3.5 \AA$ for the observation of the 001 smectic reflection and $\lambda=10 \AA$ for the observation of the small-angle region (SANS). In both cases, the multidetector-sample distance is $2 \mathrm{~m}$.

The small-angle scattering obeys the Guinier approximation for which the scattered intensity $I(\vec{q})$ is proportional to the form factor $P(\vec{q})$ associated with the main-chain part of the polymer: $1 / I(\vec{q}) \alpha 1 / P(\vec{q})=$ $N\left\langle 1+\left(q_{x}^{2} R_{x}^{2}+q_{y}^{2} R_{y}^{2}+q_{z}^{2} R_{z}^{2}\right)\right\rangle$, where $\vec{q}$ is the scattering vector $\left(|\vec{q}|=\frac{4 \pi}{\lambda} \sin \frac{\theta}{2}\right)$ with $\theta$ the scattering angle. $x, y$, or $z$ are the $O x$ axis (parallel to the velocity gradient), the $O y$ axis (parallel to the velocity direction), and the $O z$ axis (perpendicular to the velocity direction), respectively, and $N$ is the degree of polymerization.

In the smectic phase, the polymer main chains preferentially occupy the space available between the mesogenic layers [5] [Fig. 1(a)]. The main chains can be considered as already subjected to the internal constraint from the mesogen layers. By applying a mechanical shear, the viscoelastic properties of the polymer, as well as those of the liquid crystalline phase $\left(S_{A}\right)$, can be exacerbated. It is necessary to work close to the $N-S_{A}$ transition because the viscous properties of the liquid crystalline phase are rapidly reduced at low temperature.

The first series of measurements were carried out at a temperature of $93{ }^{\circ} \mathrm{C}$, slightly below the $N-S_{A}$ transition, whereas in the second series, the polymer behavior is examined during this transition. Structural information is drawn from a two-dimensional Gaussian fit of the 001 smectic reflection, whereas the conformational information is provided by a two-dimensional Lorentzian fit of the central scattering.

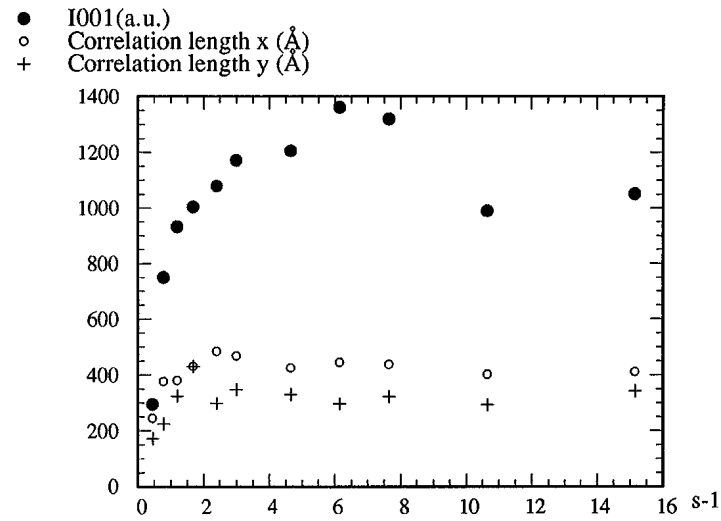

FIG. 3. Evolution of the 001 smectic reflection versus shear rate at $93{ }^{\circ} \mathrm{C}$. The points $(\bullet)$ correspond to the intensity maximum, whereas + and $\circ$ are the correlation lengths of the smectic order following $O x$ and $O y$ directions, respectively (without correction of the spectrometer resolution function).

Figure 3 presents the evolution of the intensity maximum of the 001 reflection as a function of the shear rate as well as the smectic correlation lengths in both $O x$ and $O y$ directions. The increase of both 001 intensity and correlation lengths is evidence of a shear induced alignment which drives the smectic layers parallel to the shear plane. The comparison of the correlation lengths in both directions indicates that the smectic range is higher along the velocity direction $O x$ than in the velocity gradient direction $O y$. The position of the peak is the same within $\pm 0.3 \AA$ and is independent of the shear rate, indicating that the mesogens are nearly perpendicular to the smectic plane and, on average, at the maximum possible elongation [layer thickness $(d)=$ mesogen length $\approx 29 \AA$ ].

Two regimes can be distinguished in Fig. 3 .

(i) A first regime of shear flow $\left(0<\dot{\gamma} \leq 10 \mathrm{~s}^{-1}\right)$, corresponding to the alignment process of the smectic layers parallel to the shear plane. In this regime, the 001 intensity increases rapidly and reaches a maximum for $\dot{\gamma} \approx 6-7 \mathrm{~s}^{-1}$.

(ii) A second regime corresponding to a decrease of the maximum of the 001 intensity when $\dot{\gamma}>10 \mathrm{~s}^{-1}$ (without a change of correlation lengths associated with the peak). In this regime, the shear introduces instabilities which destroy the orientation of the layers. This phenomenon is reversible (the intensity increases again once the shear is reduced) and cannot be attributed to an internal craze inside the sample since no relaxation phenomenon is observed. We conclude therefore that high shear rates act on the scale of the layers, causing them to spread randomly in the shear field [6].

Figure 4 shows the evolution of main-chain anisotropy in the vorticity plane against shear rate. SANS measurements have been alternated with diffraction measurements. The extension of the polymer main chain in the direction of velocity $R y$ becomes clearly larger when the shear increases, whereas $R x$ gets smaller and almost constant at the smallest shear rate. As we showed in our earlier paper [3], here are two simultaneous processes, a reori- 


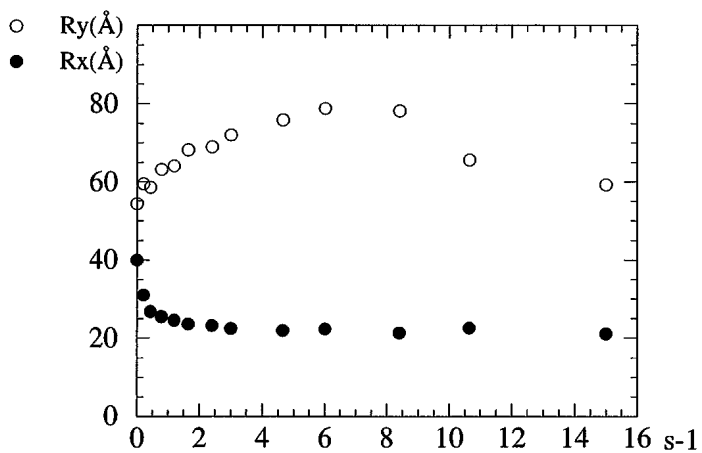

FIG. 4. Evolution of the components $R x(\bullet)$ and $R y(\circ)$ of the radius of gyration of the polymer main chain along $O x$ and $O y$ versus the shear rate at $93^{\circ} \mathrm{C}$.

entation of the smectic monodomains which takes place very early and also a progressive elongation of the main chain confined between mesogen layers, along the velocity direction $O x$. As can be ascertained from Fig. 2, the central scattering (associated to the form factor of the polymer main chain) presents no tilt but a shape elongated along $O y$. This is the signature of liquid-crystalline behavior. The difference observed for the values at rest $(R x=27 \AA, R y=36 \pm 5 \AA)$ corresponds to a partial orientation during the filling of the device. The reorientation process takes place very early since $R x$ has reached a relatively stable value when $\dot{\gamma} \approx 1 \mathrm{~s}^{-1}$. In another experiment, the polymer was previously aligned (with a magnetic field) with the smectic layers perpendicular to the shear plane. The application of a shear of $0.5 \mathrm{~s}^{-1}$ was sufficient to destroy the initial orientation and to recover the usual orientation after $\approx 100 \mathrm{~s}$. This experiment illustrates the intensity of the forces driving the smectic layers parallel to the $y 0 z$ shear plane. The value of $R x$ is smaller than the layer thickness $(R x \approx 20-25 \AA$ compared to $d \approx 29 \AA$ ) which means that the main chains do not cross the layers. This is necessary for layers to slide and could explain the decrease of molecular weight observed during the earlier shear experiment [3]. The main-chain extension in the direction parallel to the velocity $(R y)$ increases progressively and corresponds largely to the elongation process. Indeed, the sample becomes transparent as soon as a very small shear rate is applied and the polymer main chain elongates in the velocity direction with increasing shear [3]. The smaller values of $R y$, obtained at high shear rates $\left(\dot{\gamma}>10 \mathrm{~s}^{-1}\right)$, corroborate the observation carried out on the 001 reflection. The decrease of $R y$ (and probably the increase of $R x$ ) results from the disorientation of the smectic layers since the main chains are confined between the mesogen layers.

As the $N-S_{A}$ transition is approached, the relaxation time associated to the density wave of the smectic fluctuations becomes the control parameter of the dynamics of the whole system. Since polymer and liquid crystal are coupled, the polymer motion is also reduced and two critical shear rates are expected to result corresponding to both aspects. The situation described in the preceding paragraph corresponds to the case where smectic forces dominate. In order to instigate a real competition between the two behaviors, it is necessary to decrease the smectic force and hence to start the shear process $5-6^{\circ} \mathrm{C}$ above the expected $N-S_{A}$ transition to be sure to weaken the smectic forces (the nematic phase presents smectic fluctuation). It is also necessary to apply a relatively high shear since no main-chain deformation was observed in the isotropic and nematic phases [3]. We slowly decrease the temperature using the approach to the transition to induce a slowing down of the polymer motion.

The sample is heated at rest in the shear device until it reaches the isotropic phase. The shear is then progressively increased to $15 \mathrm{~s}^{-1}$, and the temperature is slowly decreased until the first diffuse intensity associated with the smectic fluctuations $\left(98^{\circ} \mathrm{C}\right)$ appears. This process is carried out on the $100 \% \mathrm{H}$ sample of $\mathrm{PMA}-\mathrm{OC}_{4} \mathrm{H}_{9}$ since a stronger smectic intensity compared to the $\mathrm{H} / \mathrm{D}$ mixture

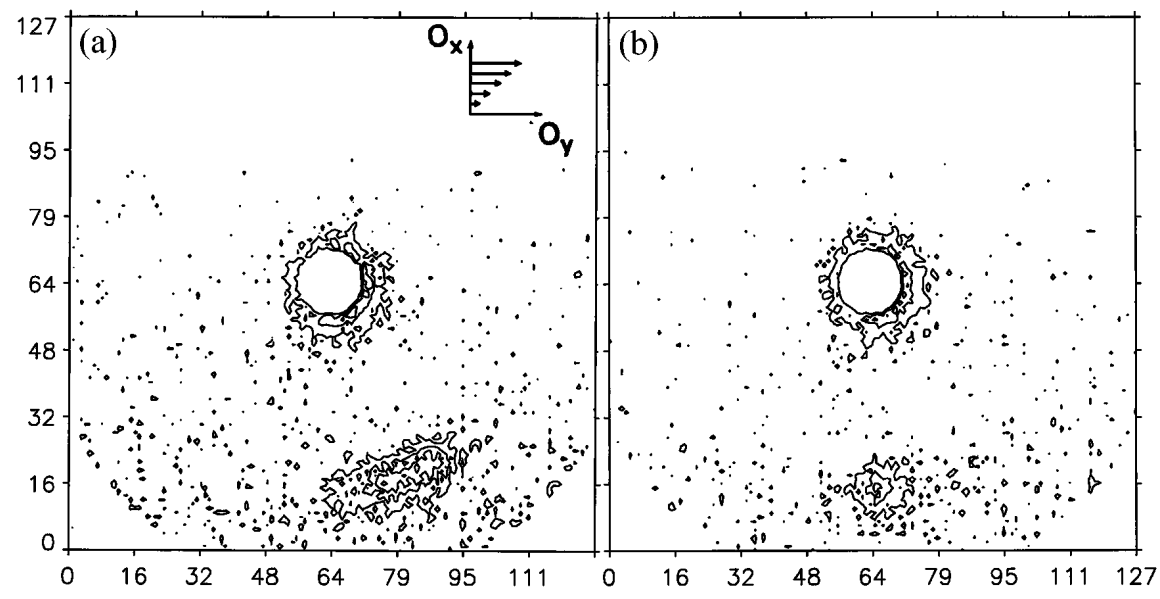

FIG. 5. Evolution of the scattering pattern obtained in the vorticity plane with the fully hydrogenated polymer submitted to a shear rate of $15 \mathrm{~s}^{-1}$ at $98^{\circ} \mathrm{C}$. The time interval between the appearance of the diffuse scattering (a) and the appearance of the 001 reflection $(\mathrm{b})$ is $\approx 1800 \mathrm{~s}$. 


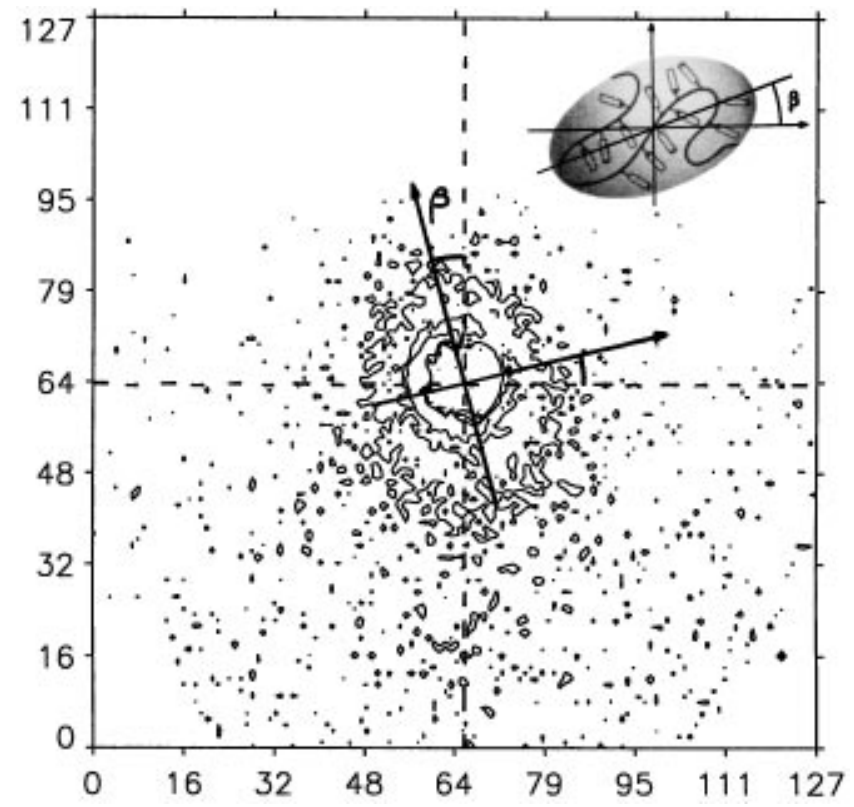

FIG. 6. Small-angle neutron scattering $(\lambda=3.5 \AA, d=$ $2 \mathrm{~m}$ ) obtained with the H/D mixture under the same experimental conditions as for Fig. 5.

and the absence of central scattering makes the measurements easier.

As can be seen in Fig. 5(a), a scattering of short correlation length $(=70 \AA)$ corresponding to the previous smectic distance $(28 \AA)$ first appears tilted at $20^{\circ}$ to the normal to the shear plane. Keeping the same temperature $\left(98 \pm 1{ }^{\circ} \mathrm{C}\right)$ and shear rate $\left(15 \mathrm{~s}^{-1}\right)$, we observe the migration of this scattering from $20^{\circ}$ to the usual stable position normal to the shear plane [Figs. 5(a) and 5(b)]. The two figures represent a $30 \mathrm{~min}$ interval.

These conditions are reproduced as faithfully as possible for the H/D mixture in order to determine the corresponding main-chain conformation. In the isotropic phase, we observe an isotropic main-chain conformation. The temperature is then slowly decreased under shear, and a slightly elongated tilted scattering (Fig. 6) appears for temperatures close to $98{ }^{\circ} \mathrm{C}$ before reaching the stable smectic phase. It corresponds to main chains tilted from $\approx 15^{\circ}$ compared to $O y$ of average dimensions $R_{x}^{*}=56 \pm$ $5 \AA$ and $R_{y}^{*}=36 \pm 5 \AA$ along the major axis. This situation recalls the "tank tread" rotation adopted by ordinary polymers under shear flow [4], indicating that the viscoelastic properties of the main chains are exacerbated. However, such a rheological behavior is not compatible with an oriented phase. This implies that the increase of the shear had induced a transition from a liquid-crystalline to a polymer-type behavior. It is very probable that the appearance of the tilted shape just above the appearance of a stable smectic phase is not by chance but is due to a slowing down of polymer motion at the outskirts of the smectic phase. Therefore, higher shear flow or higher molecular weight should produce a shear induced transition from liquid crystal to polymer behavior inside the nematic or in a weak smectic phase. The absence of mainchain deformation revealed in previous experiments [3] could be interpreted by relaxation times too short compared to the shear applied (a Deborah number $\ll 1$ ). Figures 5(a) and 5(b) indicate a return back to the ordered smectic state. This shows that the force of the smectic order increases (with time, if it is a transitional process and/ or temperature since it is controlled to within $\pm 1{ }^{\circ} \mathrm{C}$ ). A rheological behavior implying the rotation of the macromolecule is no longer possible, and the sliding of smectic layers becomes favored. This gives rise to the result shown in Fig. 5(b). The liquid-crystalline (smectic) behavior becomes predominant again.

We have demonstrated that shear can favor either a polymer or a liquid-crystalline rheological behavior; by applying the shear into the smectic phase, we observe the smectic layer alignment. This is a liquid-crystalline behavior [1]. In contrast, the application of sufficient shear above the nematic-smectic transition, with decreasing temperature, produces a tilted elongated main-chain shape. This suggests a typical polymer rheological behavior [4] and implies a transition from liquid crystalline (nematic) at rest to polymer behavior under shear flow. Then, as the smectic forces become stronger, the polymer rotation is replaced by the sliding layers which is the inverse transition, i.e., polymer to liquid crystal (smectic) behavior. Finally, our accuracy did not allow us to determine any temperature shift due to the shear. Here the rotational motion of the polymer may have an opposite influence compared to the smectic fluctuations which should increase the transition temperature under shear [7]. A cell with an improved temperature resolution would be expected to shed further light on this behavior and the parameters which control it.

We thank S. Lecommandoux and G. Kirsch for the polymer synthesis and G. Pépy for the conception of a two-dimensional data treatment.

[1] C. R. Safinya, E. B. Sirota, and R. J. Plano, Phys. Rev. Lett. 66, 1986 (1991); edited by M. Doi and S. F. Edwards (Oxford University Press, New York, 1986).

[2] R.H. Kannan, J.A. Kornfield, N. Schwenk, and C. Boeffel, Macromolecules 26, 2050 (1993).

[3] L. Noirez and A. Lapp, Phys. Rev. E 53, 1115 (1996).

[4] R. Muller, C. Picot, Y.H. Zang, and D. Froelich, Macromolecules 23, 2577 (1990).

[5] P. Davidson and A. M. Levelut, Liq. Cryst. 11, 469 (1992); H. G. Ohm, R. G. Kirste, and R.C. Oberthür, Makromol. Chem. Theory Simul. 116, 1387 (1988)L. Noirez, P. Davidson, W. Schwarz, and G. Pépy, Liq. Cryst. 16, 1081 (1994).

[6] L. Noirez and A. Lapp, Physica B (Amsterdam) (to be published).

[7] P. C. Hohenberg and B. I. Halperin, Rev. Mod. Phys. 49, 435 (1977); R. F. Bruinsna and C. R. Safinya, Mater. Res. Soc. Symp. Proc. 177, 153 (1990). 